\section{PTU-056 LOSS OF CATHELICIDIN (LL-37) IS ASSOCIATED WITH COLORECTAL CANCER PROGRESSION}

${ }^{1}$ Ross J Porter*, 'Graeme I Murray, ${ }^{2} \mathrm{Ji}$ M Wang, ${ }^{3}$ Teizo Yoshimura, ${ }^{1}$ Mairi H McLean. ${ }^{1}$ School of Medicine, Medical Sciences and Nutrition, University of Aberdeen, UK; ${ }^{2}$ Cancer and Inflammation Program, Center for Cancer Research, National Cancer Institute at Frederick, USA; ${ }^{3}$ Department of Pathology and Experimental Medicine, Graduate School of Medicine, Dentistry and Pharmaceutical Sciences, Okayama University, Japan

\subsection{6/gutjnl-2018-BSGAbstracts.397}

Introduction Cathelicidin (LL-37) is an innate anti-microbial peptide. Previous in vitro studies suggest a protective role in colonic carcinogenesis. Our recent pre-clinical data revealed that genetic knock out of cathelicidin led to increased size and number of colorectal tumours in the azoxymethane mediated murine model of colorectal cancer (CRC). Cathelicidin expression has not been characterised in human colorectal cancer.

Methods Intensity of epithelial cytoplasmic LL-37 was assessed immunohistochemically in a tissue microarray representing 650 colorectal cancers and 50 paired normal colorectal mucosa samples. Tissue was obtained from chemotherapy and radiotherapy naïve patients obtained at time of surgery for primary colonic cancer, sourced from the Grampian Tissue Biorepository. Ethical approval was granted by the Grampian Biorepository Scientific Access Group. Clinico-pathological data were available for each case, including survival up to 18.2 years post-resection. Expression intensity of LL-37 was independently assessed by two observers, blind to clinico-pathological data, as absent, weak, moderate or strong. Descriptive analysis, $\chi^{2}$ test, Fisher's exact test and log-rank survival analysis was performed using IBM SPSS Statistics (Version 24.0.0.0).

Results The expression of cytoplasmic LL-37 was weaker in colorectal cancer compared to normal colonic epithelium $(\mathrm{p}<0.001)$. Increased intensity of LL-37 expression was present in patients staged Dukes A compared to Dukes B $(p=0.004)$ or Dukes C $(p=0.003)$. There was no correlation of LL-37 expression to tumour site, differentiation, extramural venous invasion or mismatch repair protein status. Normal colonic mucosa from patients with Dukes A CRC expressed stronger cytoplasmic LL-37 compared to normal colonic mucosa from patients with Dukes C CRC $(p=0.031)$. There was no relationship between LL-37 expression and overall survival.

Conclusions Loss of epithelial cytoplasmic LL-37 is associated with progression of colorectal cancer, confirming translation of pre-clinical data to human disease. There may be a global field change in LL-37 expression in distant non-malignant cells as CRC progresses. The functional impact of this warrants further investigation and may reveal new insight into the pathogenesis of colorectal cancer.

\section{PTU-057 REFERRALS FOR SUSPECTED COLORECTAL CANCER BEFORE AND AFTER THE UPDATED NICE GUIDELINES IN JUNE 2015}

Victoria Proctor*, Ioana Iftimie-Nastase, Jessica Sinclair, Rangasamy Sivakumar, Syed Shah. Mid Yorkshire Hospitals, Wakefield, UK

\subsection{6/gutjnl-2018-BSGAbstracts.398}

Introduction The NICE guideline for referral to specialist services with suspected colorectal cancer (CRC) was last updated in June 2015, with expansion of the criteria. This study aimed to compare referral patterns and clinical practice, in terms of the investigations performed, over two years before (01/06/ $2013-31 / 05 / 2015)$ and after (01/06/2015-31/05/2017) introduction of the updated guideline.

Methods A retrospective study of all patients referred to our unit with a suspected CRC in the two years before and two years after introduction of the updated guideline compared referral rates, the number of colorectal and non-colorectal (non-CRC) cancers, the TNM stage of CRC and the investigations performed.

Results The total number of referrals increased from 6075 to 7951, with the rate of CRC decreasing from 5.14\% to 5.01\% $(p=0.08)$. The number of non-CRCs increased from $1.17 \%$ to $1.61 \%(p=0.02)$. There was no significant difference in the TNM stage of CRC (figure 1).

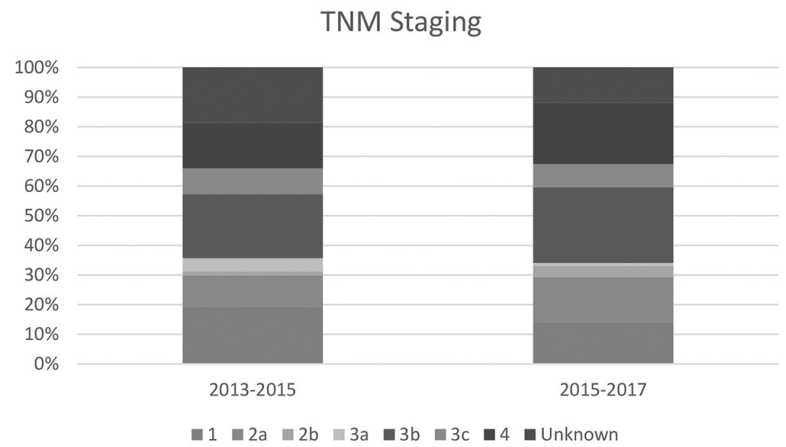

Abstract PTU-057 Figure 1 TNM staging of detected colorectal cancers

The use of flexible sigmoidoscopy $(17.01 \%$ vs. $28.52 \%$, $\mathrm{p}=0.002)$ and CTC (9.54\% vs. $17.78 \%, \mathrm{p}=0.007)$ increased and the use of colonoscopy decreased $(81.33 \%$ vs. $67.78 \%$, $\mathrm{p}=0.0006)$. The use of CT $(91.29 \%$ vs $92.59 \%)$ and MRI scanning was not significantly different between groups (figure 2).

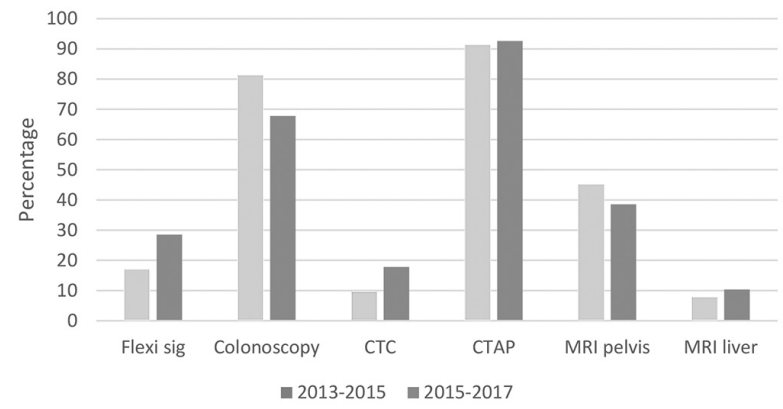

Abstract PTU-057 Figure 2 Investigations performed for detected colorectal cancers

Conclusions CTC was more common in the later cohort, likely related to increasing availability of this imaging modality. This may also explain the increase in flexible sigmoidoscopy, as left sided lesions on CTC require flexible sigmoidoscopy only, rather than full colonoscopy.

Expansion of the referral criteria has not significantly impacted on the number of new CRCs, but has increased the number of non-CRCs. In those presenting with symptoms such as anaemia, abdominal pain and weight loss, with an absence of specific lower gastrointestinal symptoms, CT would be a more appropriate initial investigation than luminal investigations. This will aid in the diagnosis of other significant pathologies, including the many non-CRCs detected via this referral pathway, whilst minimising exposure to invasive luminal investigations with their associated morbidity. 